\title{
Antithrombin deficiency in pregnancy
}

\author{
Shivani Durai, Lay Kok Tan, Serene Lim
}

Department of Obstetrics \& Gynaecology, Singapore General Hospital, Singapore, Singapore

\section{Correspondence to} Dr Shivani Durai, dr.shivani.durai@gmail.com

Accepted 3 May 2016

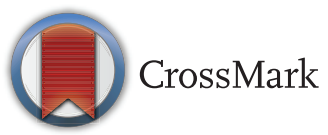

To cite: Durai S, Tan LK Lim S. BMJ Case Rep Published online: [please include Day Month Year] doi:10.1136/bcr-2015213310

\begin{abstract}
SUMMARY
We present a case of a 39-year-old, gravida 3 para 2, Chinese female with a history of inherited type 1 Antithrombin deficiency and multiple prior episodes of venous thromboembolism. She presented at 29 +4 weeks' gestation with severe pre-eclampsia complicated by haemolysis, elevated liver enzymes and low platelet (HELLP) syndrome. She subsequently underwent an emergency caesarean section for nonreassuring fetal status, which was complicated by postpartum haemorrhage secondary to uterine atony, requiring a B-Lynch suture intraoperatively.
\end{abstract}

\section{BACKGROUND}

Venous thromboembolism (VTE) remains a major cause of direct maternal deaths in the world, afflicting $0.5-3.0$ per 1000 pregnancies. $^{1} \mathrm{~A}$ physiological increase in clotting factors and an expanding gravid uterus compressing on adjacent pelvic veins, increases the thrombotic risk of pregnancy by 10 fold. $^{2}$ The presence of inherited thrombophilia such as Antithrombin (AT) deficiency is an additional risk factor for maternal thromboembolism. ${ }^{3}$ The risk of developing a VTE in this population is up to $50 \%{ }^{4}$

The association between inherited thrombophilia and placenta-mediated complications such as preeclampsia is still being studied. Pre-eclampsia is a major contributor to maternal and perinatal mortality. It has been estimated that $40 \%$ of the patients who present with pre-eclampsia have an underlying thrombophilia. ${ }^{5}$ These patients with an associated thrombophilia such as AT deficiency also appear to be at increased risk of haemolysis, elevated liver enzymes and low platelet (HELLP) syndrome. ${ }^{5}$

There are many studies on inherited thrombophilias in pregnancy, especially within the Caucasian population, where the prevalence of the disease is more common. In contrast, there is a lack of local data on inherited thrombophilias, with no published studies addressing the association of thrombophilia and placenta-related outcomes in the local obstetric population in Singapore.

We report a case of a patient with severe preeclampsia and HELLP syndrome in the setting of inherited AT deficiency.

\section{CASE PRESENTATION}

A 39-year-old, gravida 3 para 2, Chinese female with AT deficiency was transferred to our unit at $29+4$ weeks' gestation for further management of severe pre-eclampsia.

Twelve years earlier, she had collapsed at an airport due to cardiopulmonary arrest from a rightsided pulmonary embolism secondary to a popliteal deep vein thrombosis (DVT) following a long-haul flight. She had also just started the combined oral contraceptive pill (COCP) 5 days earlier when the incident occurred. Ventilation/perfusion scanning demonstrated matched ventilation and perfusion deficits bilaterally with extensive involvement of the right lower lobe resembling pulmonary infarctions. She required 5 days of ventilator and ionotropic support in an intensive care unit (ICU) overseas. She was diagnosed with inherited type 1 AT deficiency following an extensive haematological workup (which included a diagnostic AT functional assay) supported by a strong familial history of VTE affecting her father, paternal aunt and cousin. She was started on warfarin on her discharge following a 2-week hospital stay. On her return to Singapore, she continued her follow-up with a private haematologist.

Three years following the event, she had an uneventful pregnancy and vaginal birth of her first child while on nadroparin (a low-molecular-weight heparin (LMWH)). Five years later, she had another uneventful vaginal delivery of her second child while on tinzaparin (a different LMWH). However, 4 months following her delivery, she developed an extensive right lower limb DVT involving her common femoral and popliteal vein while on warfarin for anticoagulation postnatally. She was counselled against another pregnancy by both her obstetrician and haematologist. However, having fallen pregnant unexpectedly 5 years later, she was very keen to keep her pregnancy.

During this pregnancy, she received her antenatal care in the private sector. She developed a DVT with complete thrombosis of her left superficial femoral vein extending distally to her popliteal, peroneal and posterior tibial veins at 12 weeks' gestation. A repeat ultrasound of her left lower limb showed resolution of her VTE. She was also started on methyldopa from 20 weeks' gestation for pregnancy-induced hypertension (PIH) with a previously normal antenatal booking blood pressure. This was subsequently switched to long-acting nifedipine due to a suboptimal response in her blood pressure.

At $29+1$ weeks, she was admitted for preeclampsia for worsening hypertension and newonset proteinuria. She was subsequently transferred to our tertiary hospital 3 days later for severe refractory hypertension secondary to pre-eclampsia. At the time of transfer, her blood pressure was elevated at $197 / 123 \mathrm{~mm} / \mathrm{Hg}$ and she was reporting epigastric discomfort. She was noted to have hyperreflexia but no clonus. Cardiotocography (CTG) and an ultrasound showed reassuring fetal status.

She was started on intravenous labetalol and magnesium sulfate for eclampsia prophylaxis. 
A course of betamethasone was administered for acceleration of fetal lung maturity and she was placed on continuous CTG monitoring. The patient and her partner were also counselled by the neonatal team regarding the complications of prematurity in infants.

\section{INVESTIGATIONS}

Six hours later, there was new evidence of HELLP syndrome diagnosed from repeat laboratory studies demonstrating elevations in liver enzymes (Alkaline Phosphatase (ALP): $224 \mathrm{U} / \mathrm{L}$, Alanine Transaminase (ALT): $139 \mathrm{U} / \mathrm{L}$, Aspartate Transaminase (AST): $112 \mathrm{U} / \mathrm{L}$, albumin: $26 \mathrm{~g} / \mathrm{L}$ ) and a reduction in platelet count from 247000 to 61000 on a background of a normal haematocrit. Her lactate dehydrogenase level was elevated at $1084 \mathrm{U} / \mathrm{L}$ and haptoglobin level was reduced at $<0.10 \mathrm{~g} / \mathrm{L}$, with a normal coagulation profile. Her uric acid level was noted to be normal at $289 \mathrm{umol} / \mathrm{L}$.

\section{TREATMENT}

She underwent an emergency caesarean section (CS) at this stage as there was also CTG evidence of non-reassuring fetal status evidenced by reduced variability and unprovoked shallow decelerations.

Intraoperatively, clear liquor was noted with no signs of abruption. However, the uterus was atonic despite uterine massage and the administration of multiple uterotonics including carbetocin, intramuscular carboprost and a continuous intravenous oxytocin drip. Placement of a B-lynch suture was finally successful in compressing the uterus and achieving haemostasis. As the patient was not keen for for further children, tubal ligation was also performed. The total estimated blood loss during surgery was $500 \mathrm{~mL}$ with placental histology confirming pre-eclampsia.

\section{OUTCOME AND FOLLOW-UP}

A baby boy weighing $1265 \mathrm{~g}$ was delivered at 29+5 weeks' gestation with Apgar scores of 2 and 8 at 1 and $5 \mathrm{~min}$, respectively. The baby was intubated and transferred to the neonatal intensive care unit where he remained for 12 days due to apnoea of prematurity. Cord blood gas indicated an arterial $\mathrm{pH}$ of 7.28 with a base excess of 1.3 and a venous $\mathrm{pH}$ of 7.30 with a base excess of 1.1. He was discharged well at a corrected age of $34+5$ weeks.

Postoperatively, the patient's blood pressure was well controlled with oral antihypertensives and her liver function and platelet count normalised. Her stay was, however, complicated by a postoperative ileus, which resolved with conservative management and she was discharged well 6 days post her admission. Tinzaparin was restarted within 6 hours of her CS and eventually converted to warfarin on her ninth postoperative day.

\section{DISCUSSION}

AT is an essential natural anticoagulant that inhibits thrombin and factor Xa (figure 1). ${ }^{7}$ AT circulates in plasma in a physiologically less active form. Its anticoagulation effect is amplified in the presence of heparin which is present on vascular endothelium or can be given exogenously. It also functions as a cofactor in the binding of AT to thrombin, forming AT-clotting factor complexes that are swiftly removed from the circulation. ${ }^{7}$

Type I AT deficiency results in a quantitative reduction of functionally normal AT while type II AT deficiency results in the production of a qualitatively abnormal protein. There is a higher risk of thrombosis associated with the type I variety. ${ }^{8} 9$

Type II AT deficiency can be further subdivided into the less common but more thrombogenic type IIa variant and the more common but less thrombogenic type IIb variant. ${ }^{10}$ Type IIa deficiency is caused by mutations in the thrombin-binding site while type IIb deficiency is caused by a defect in the heparin-binding region of AT. $^{10}$

There are at least 256 mutations in the AT gene causing various phenotypes. ${ }^{11}$ These mutations are distributed throughout the AT molecule. Reactive centre defects have the greatest potential for thrombosis and heparin-binding defects have the least. ${ }^{11}$ The complete absence of AT is lethal.

However, few published cases of patients with homozygous mutations in the heparin-binding site (HBS) have suggested that homozygosity results in an earlier presentation of thrombotic disease. ${ }^{12}$ Moreover, the replacement of threonine-85 (Thr-85) methionine (known as antithrombin-Wibble) results in a mild adult-onset thrombotic disease, whereas replacement of the same Thr- 85 by lysine (known as antithrombin-Wobble) results in early childhood onset of thrombosis. ${ }^{12}$

Alternatively, acquired AT deficiency is predominantly due to consumption. It is seen when activation of the coagulation system is abnormal. It is noted in conditions such as disseminated intravascular coagulation (DIC), nephrotic syndrome and microangiopathic haemolytic anaemias. ${ }^{6}$

\section{Thrombophilia and placenta-mediated complications in pregnancy}

Pre-eclampsia has been widely studied, however, its cause remains elusive. It is believed to occur due to impaired
Figure 1 Illustration of the action of antithrombin. ${ }^{6}$

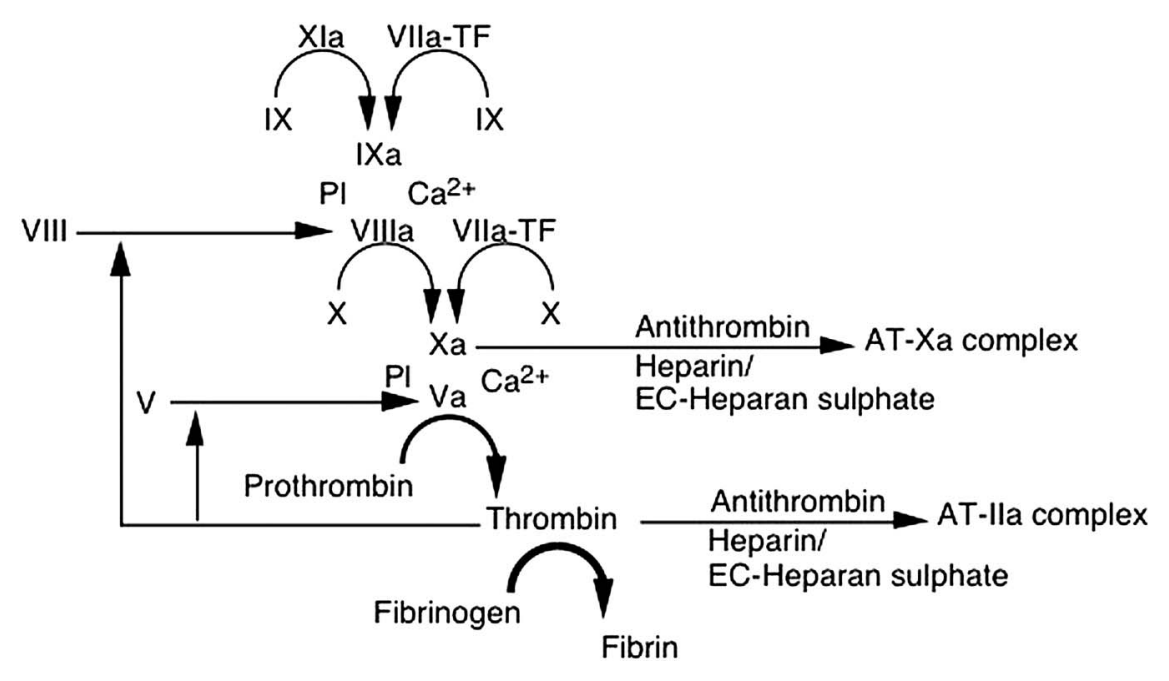

Durai S, et al. BMJ Case Rep 2016. doi:10.1136/bcr-2015-213310 
placentation, placental ischaemia, vascular endothelial damage, platelet dysfunction and alterations in the coagulation cascade. ${ }^{13}$ The thrombotic risk associated with inherited thrombophilia has been hypothesised to be the underlying cause of pre-eclampsia.

However, thrombosis is unlikely to be the only mechanism by which thrombophilia increases the risk of pregnancy complications. Trophoblast differentiation and early placentation may also be involved through yet unidentified mechanisms. ${ }^{14}$

Although most data surrounding the relationship between thrombophilia and adverse pregnancy outcomes are obtained from studies in women with antiphospholipid syndrome (APLS), some research also suggests an association between adverse pregnancy outcomes and hereditary thrombophilias such as AT deficiency. ${ }^{15}$ However, there is still no clear evidence to suggest that thrombophilias cause placenta-mediated pregnancy complications such as pregnancy loss, pre-eclampsia, intrauterine growth restriction and placental abruption. A recent large systematic review of prospective cohort studies highlighted that the association between thrombophilia and these placentamediated pregnancy complications is still far from proven. ${ }^{16}$

Another large multicentre cohort study, known as the Montreal Preeclampsia Study, also did not find evidence to support an association between inherited thrombophilia and increased risk of pre-eclampsia. It stated that placental underperfusion was associated with pre-eclampsia, but it did not appear to be secondary to thrombophilia. ${ }^{17}$

Studies such as the TIPPS trial also report that antepartum prophylactic LMWH, specifically dalteparin, does not reduce the occurrence of VTE, pregnancy loss or placenta-mediated pregnancy complications in pregnant women with thrombophilia, and is in fact associated with an increased risk of minor bleeding. ${ }^{18}$

\section{Management of AT deficiency in pregnancy}

The Royal College of Obstetricians and Gynaecologists (RCOG) guidelines state that women with recurrent VTE associated with AT deficiency require thromboprophylaxis with higher dose LMWH, either prophylactic (12-hourly) or weight-adjusted (75\% of treatment dose) antenatally and for 6 weeks post partum or until converted back to warfarin after delivery, when the risk of haemorrhage is low. ${ }^{19}$ In view of our patient's history of recurrent VTE, she will be required to be on lifelong anticoagulation on warfarin. ${ }^{20}$ It is also highly recommended by the RCOG that these patients are managed by haematologists who are specialists in treating thrombophilia in pregnancy. ${ }^{21}$

\section{Intrapartum management}

The College of Obstetricians and Gynaecologists Singapore recommends the cessation of LMWH when labour begins. ${ }^{22}$ It also advises that LMWH can be administered 3 hours after atraumatic epidural or spinal anaesthesia or removal of an epidural catheter. Patients should also be warned to discontinue self-injections of LMWH as soon as they believe themselves to be in labour, and not restart until evaluation in hospital. ${ }^{22}$

\section{Postpartum prophylaxis}

Post partum, the first dose of LMWH should be given 6 hours after delivery when haemostasis is secured and after removal of the epidural catheter. $0.2 \mathrm{mLs}$ of Nadroparin can be given if the patient's body weight is below $50 \mathrm{~kg}$ or $0.3 \mathrm{mLs}$ can be given if the patient's body weight exceeds $50 \mathrm{~kg}$. Alternatively, enoxaparin can be used at a $40 \mathrm{mg}$ daily dose. ${ }^{22}$

If the patient prefers not to continue self-injecting, LMWH can be replaced by warfarin starting on the first or second postpartum day. ${ }^{22}$ The LMWH can be withdrawn when the international normalised ratio (INR) has been within the target range of 2-3 for two consecutive days. It is advisable to maintain the INR at 2 for 6 weeks post partum. There is no contraindication to breast feeding when the mother is being treated with LMWH, warfarin or other coumarins. ${ }^{22}$

In some patients with AT deficiency who need heparin therapy, AT concentrates may have to be given so that heparin can work optimally. Heparin (including LMWH) may not thin the blood optimally if an individual has low AT levels. This is because heparin's effect depends on the presence of AT. In such instances, a higher heparin dose may need to be given. In some situations, a patient can be 'heparin resistant' and will not respond effectively to heparin at all. It is in these circumstances that treatment with intravenous AT concentrates can be considered. ${ }^{23}$

\section{Screening for thrombophilias in complicated pregnancies}

Screening for thrombophilia in pregnancy remains controversial, especially as randomised controlled trials have not shown that use of anticoagulants during pregnancy prevents pre-eclampsia in women who are diagnosed with thrombophilia. ${ }^{24}$ The TREAT study found that if thromboprophylaxis is given to women who are thrombophilia positive, selective screening for medical and/or family history of VTE is more cost-effective than universal screening of pregnant patients. ${ }^{24}$ The Multiple Environmental and Genetic Assessment (MEGA) study also showed that testing for inherited thrombophilia did not reduce recurrence of venous thrombosis. ${ }^{25}$

The American College of Obstetricians and Gynecologists (ACOG) guidelines state that there is insufficient evidence to either screen or treat women with inherited thrombophilias and obstetric history that includes complications such as pre-eclampsia. ${ }^{25}$

This is further supported by the British Committee for Standards in Haematology (BCSH) Guidelines, which states that antithrombotic therapy should not be given to pregnant women on the basis of investigations for heritable thrombophilia. ${ }^{26}$ Further stating, that randomised controlled trials are still currently underway. It currently does not recommend antithrombotic therapy for women with heritable thrombophilias as there have been no proven benefits as of yet. ${ }^{26}$

\section{AT replacement}

Plasma human AT concentrates (phAT) and recombinant human AT (ATryn) are the two types of commercial AT concentrates that currently exist. No randomised clinical trials have been performed in determining their need and effectiveness in pregnancy. No guidelines or consensus statements exist as to when AT concentrates should be used.

Despite full anticoagulation during pregnancy and the postpartum period, women with AT deficiency are still susceptible to developing VTE, particularly during childbirth, when anticoagulation is withheld. There is uncertainty if AT replacement therapy during this period would be a useful alternative.

In a case series, where six women with AT deficiency and intrapartum DVTs were treated with plasma-derived AT concentrate (pdAT), none of the six patients experienced recurrent thrombosis while receiving treatment with AT concentrate. ${ }^{27}$

In the phase III trial leading to approval of ATryn in the USA, antenatal patients required frequent dose modifications. However, a case series of three AT deficient women treated with LMWH during pregnancy and ATryn at delivery asserted that a continuous infusion of AT avoided peak and trough levels. All three patients had uneventful deliveries. ${ }^{28}$ 
Limited studies have shown the potential for AT therapeutic treatment of women with pre-eclampsia. The PRESERVE-1 trial to assess whether ATryn prolongs pregnancy in mothers with early-onset pre-eclampsia is currently underway.

Studies have been inconclusive in many of the associations between thrombophilias and adverse pregnancy outcomes.

Routine laboratory screening for thrombophilias such as AT deficiency may not yield important therapeutic benefits, as these conditions are very rare in the general population and even less likely to be associated with modifiable adverse pregnancy outcomes.

Women with a history of spontaneous VTE or first-degree relatives with a significant clotting history should be considered for thrombophilia testing. An early diagnosis of thrombophilia with regular obstetric and haemotological follow up will aid in the prevention of maternal and fetal complications.

AT replacement therapies are yet to be further evaluated, especially with evidence of isolated case studies showing promising results. It may also be a useful adjunct in the treatment of preeclampsia in women with AT deficiency.

Although the large multicentre, randomised TIPPS trial suggests that the occurrence of VTE, pregnancy loss or placentamediated pregnancy complications in pregnant women with thrombophilia is not reduced with LMWH anticoagulation, the use of LMWH was warranted in our patient. The higher thrombogenic potential of AT deficiency, her strong history of multiple previous VTEs and the subsequent development of severe pre-eclampsia and HELLP syndrome during her pregnancy justified the use of LMWH.

Had she not opted for postpartum sterilisation, she would have had to be meticulously counselled on the future risk of placenta-mediated pregnancy complications, her VTE risk of up to $70 \%$ and the need for LMWH and close surveillance in all her future pregnancies.

\section{Learning points}

- Antithrombin (AT) deficiency is a rare autosomal dominant condition and is the most thrombogenic of all hereditary thrombophilias.

- The link between AT deficiency and pre-eclampsia is still being investigated.

- There are two main types of AT deficiency; type I AT deficiency results in a quantitative reduction in functionally normal AT, while type 2 AT deficiency is the result of producing functionally abnormal antithrombin.

- Women with AT deficiency and a history of venous thromboembolism (VTE) are at particular risk of recurrent VTEs during pregnancy. It is recommended that they are started on thromboprophylaxis throughout their pregnancy.

Competing interests None declared.

Patient consent Obtained.

Provenance and peer review Not commissioned; externally peer reviewed.

\section{REFERENCES}

1 Snow V, Qaseem A, Barry P, et al, American College of Physicians, American Academy of Family Physicians Panel on Deep Venous Thrombosis/Pulmonary Embolism. Management of venous thrombo-embolism: a clinical practice guideline from the American College of Physicians and the American Academy of Family Physicians. Ann Intern Med 2007;146:204-10.
2 Pabinger I, Grafenhofer $\mathrm{H}$, Kyrle PA, et al. Temporary increase in the risk for recurrence during pregnancy in women with a history of venous thromboembolism. Blood 2002;100:1060-2.

3 Vucić N, Frleta M, Petrović D, et al. Thrombophilia, preeclampsia and other pregnancy complications. Acta Med Croatica 2009;63:297-305.

4 Duhl AJ, Paidas MJ, Ural SH, et al. Antithrombotic therapy and pregnancy: consensus report and recommendations for prevention and treatment of venous thromboembolism and adverse pregnancy outcomes. Am J Obstet Gynecol 2007; 197:457.e1-21

5 Brenner B, Lanir N, Thaler I. HELLP syndrome associated with factor V R506Q mutation. Br J Haematol 1996;92:999-1001.

6 A Practical Guide to Laboratory Haemostasis. http://www.practical-haemostasis.com/ Thrombophilia\%20Tests/at assays.html

7 Kottke-Marchant K, Duncan A. Antithrombin deficiency: issues in laboratory diagnosis. Arch Pathol Lab Med 2002;126:1326-36.

8 Tait RC, Walker ID, Perry DJ, et al. Prevalence of antithrombin deficiency in the healthy population. Br J Haematol 1994;87:106-12.

9 Wells PS, Blachman MA, Henderson P, et al. Prevalence of anti thrombin deficiency in healthy blood donors: a cross sectional study. Am J Hematol 1994;45:321-4.

10 Patnaik MM, Moll S. Inherited antithrombin deficiency: a review. Haemophilia 2008;14:1229-39.

11 Wickstrom K, Edelstam G, Lowbeer $\mathrm{CH}$, et al. Reference intervals for plasma levels of fibronectin, von Willebrand factor, free protein $S$ and antithrombin during third-trimester pregnancy. Scand J Clin Lab Invest 2004;64:31-40.

12 Sarper N, Orlando C, Demirsoy U, et al. Homozygous antithrombin deficiency in adolescents presenting with lower extremity thrombosis and renal complications: two case reports from Turkey. J Pediatr Hematol Oncol 2014;36:e190-2.

13 Lain KY, Roberts JM. Contemporary concepts of the pathogenesis and management of preeclampsia. JAMA 2002;287:3183-6.

14 Kamphuisen PW, Lensen R, Houwing-Duistermaat JJ, et al. Heritability of elevated factor VIII antigen levels in factor V Leiden families with thrombophilia. $\mathrm{Br} J$ Haematol 2000;109:519-22.

15 Rodger MA, Walker MC, Smith GN, et al. Is thrombophilia associated with placenta-mediated pregnancy complications? A prospective cohort study. J Thromb Haemost 2014;12:469-78.

16 Rodger MA, Paidas M, McLintock C, et al. Inherited thrombophilia and pregnancy complications revisited. Obstet Gynecol 2008;112(Pt 1):320-4.

17 Susan RK, Robert P, Helen M, et al. Inherited thrombophilia and preeclampsia within a multicenter cohort: the Montreal Preeclampsia Study. Am J Obstet Gynecol 2009;200:151.e1-9.

18 Rodger MA, Hague WM, Kingdom J, et al. Antepartum dalteparin versus no antepartum dalteparin for the prevention of pregnancy complications in pregnant women with thrombophilia (TIPPS): a multinational open-label randomised trial. Lancet 2014:384:1673-83.

19 Bates SM, Greer IA, Pabinger I, et al. Venous thromboembolism, thrombophilia, antithrombotic therapy, and pregnancy: American College of Chest Physicians Evidence-Based Clinical Practice Guidelines (8th edition). Chest 2008;133: 844S-6S.

20 Royal College of Obstetricians and Gynaecologists (RCOG). Thromboembolic disease in pregnancy and the puerperium: acute management. London, UK: Royal College of Obstetricians and Gynaecologists (RCOG), 2007:17 p. (Green-top guideline; no. 28).

21 Pomp ER, Lenselink AM, Rosendaal FR, et al. Pregnancy, the postpartum period and prothrombotic defects: risk of venous thrombosis in the MEGA study. J Thromb Haemost 2008;6:632-7.

22 Clinical Practice Guideline on DVT Prophylaxis in Obstetrics and Gynaecology, College of Obstetricians and Gynaecologists Singapore 2006.

23 Lemmer JH, Despotis GJ. Antithrombin III concentrate to treat heparin resistance in patients undergoing cardiac surgery. J Thorac Cardiovasc Surg 2002;123:213-17.

24 Wu O, Robertson L, Twaddle S, et al. Screening for thrombophilia in high-risk situations: systematic review and cost-effectiveness analysis. The Thrombosis: Risk and Economic Assessment of Thrombophilia Screening (TREATS) study. Health Technol Assess 2006;10:1-110.

25 American College of Obstetricians and Gynecologists (ACOG). Inherited thrombophilias in pregnancy. Washington DC: American College of Obstetricians and Gynecologists (ACOG), 2013:12 p. (ACOG practice bulletin; no. 138).

26 Baglin T, Gray E, Greaves M, et al, British Committee for Standards in Haematology. Clinical guidelines for testing for heritable thrombophilia. $\mathrm{Br} J$ Haematol 2010;149:209-20.

27 Kreuziger LMB, Prosen TL, Reding MT. Use of recombinant human antithrombin concentrate in pregnancy. Int J Womens Health 2013;5:583-6.

28 Tanimura K, Ebina Y, Sonoyama A, et al. Argatroban therapy for heparin-induced thrombocytopenia during pregnancy in a woman with hereditary antithrombin deficiency. J Obstet Gynaecol Res 2012;38:749-52. 
Copyright 2016 BMJ Publishing Group. All rights reserved. For permission to reuse any of this content visit http://group.bmj.com/group/rights-licensing/permissions.

BMJ Case Report Fellows may re-use this article for personal use and teaching without any further permission.

Become a Fellow of BMJ Case Reports today and you can:

- Submit as many cases as you like

- Enjoy fast sympathetic peer review and rapid publication of accepted articles

- Access all the published articles

- Re-use any of the published material for personal use and teaching without further permission

For information on Institutional Fellowships contact consortiasales@bmjgroup.com

Visit casereports.bmj.com for more articles like this and to become a Fellow 